\title{
Quick and dirty methods for studying black-hole resonances.
}

\author{
Kostas Glampedakis \\ Department of Physics and Astronomy, Cardiff University P.O. Box 913, Cardiff, CF24 3YB, UK* \\ Nils Andersson \\ Department of Mathematics, University of Southampton, Southampton SO17 1BJ, UK
}

(Dated: October 25, 2018)

\begin{abstract}
We discuss simple integration methods for the calculation of rotating black hole scattering resonances both in the complex frequency plane (quasinormal modes) and the complex angular momentum plane (Regge poles). Our numerical schemes are based on variations of "phase-amplitude" methods. In particular, we discuss the Prüfer transformation, where the original (frequency domain) Teukolsky wave equation is replaced by a pair of first-order non-linear equations governing the introduced phase functions. Numerical integration of these equations, performed along the real $r_{*}$-axis (where $r_{*}$ denotes the usual tortoise radial coordinate), or along rotated contours in the complex $r_{*}-$ plane, provides the required $\mathcal{S}$-matrix element (the ratio of amplitudes of the outgoing and ingoing waves at infinity). Müller's algorithm is then employed to conduct searches in the complex plane for the poles of this quantity (which are, by definition, the desired resonances). We have tested this method by verifying known results for Schwarzschild quasinormal modes and Regge poles, and provide new results for the Kerr black hole problem. We also describe a new method for estimating the "excitation coefficients" for quasinormal modes. The method is applied to scalar waves moving in the Kerr geometry, and the obtained results shed light on the long-lived quasinormal modes that exist for black holes rotating near the extreme Kerr limit.
\end{abstract}

\section{INTRODUCTION}

During the last few decades it has become clear that resonant phenomena play an important role in black hole physics [1]. Numerical experiments, pioneered by Vishveshwara 2] more than thirty years ago, and continued since then by many other authors, clearly show the dominance of the so-called quasinormal mode (QNM) oscillations in most dynamical processes involving black holes. The importance of this phenomenon is further emphasised by the possibility that future observations of QNM "ringing" following, say, the merger of two black holes by the network of interferometric gravitational wave detectors may provide direct identification of black holes. This exciting prospect has motivated a large number of investigations into the nature of the QNMs. An exhaustive list of papers on the subject can be found in Refs. 3, 4].

The spectrum of QNM frequencies has been studied extensively in the literature, especially for the Schwarzschild black hole. It is well-known that the actual mode calculation is a non-trivial task. Recall that any physically acceptable solution to the relevant (homogeneous) wave equation takes the form of a purely "ingoing" wave $\sim \exp \left(-i \omega r_{*}\right)$ (where $r_{*}$ is the tortoise radial coordinate) at the event horizon and a mixture of "ingoing/outgoing" waves $\sim$ $A_{\text {in }} \exp \left(-i \omega r_{*}\right)+A_{\text {out }} \exp \left(i \omega r_{*}\right)$ far away from the black hole (such solutions exist because the effective curvature potential falls off asymptotically towards both the horizon and infinity, i.e. when $r_{*} \rightarrow \pm \infty$ ). A QNM solution corresponds to purely outgoing waves at infinity. In other words, to search for a QNM one must first calculate the asymptotic amplitudes $A_{\text {out }}$ and $A_{\text {in }}$, and then try to find a frequency that satisfies the required QNM boundary condition $A_{\text {in }}=0$. However, direct numerical integration of the wave equation leads to serious numerical problems. Since the QNMs are expected to be damped due to gravitational-wave emission the corresponding solutions must grow exponentially as one approaches infinity and the horizon (on a $t$-constant hypersurface). In order to identify a QNM one must be able to filter out the solution that decays exponentially at infinity. In other words, the numerical calculation requires exponential precision.

Since the first "direct" calculation of the slowest damped Schwarszchild QNMs by Chandrasekhar and Detweiler [5] various alternative schemes have been devised. Detweiler [6] was able to determine the fundamental Kerr QNM with adequate accuracy by examining the functional form of the asymptotic amplitudes along the real frequency axis. Ferrari and Mashhoon [] exploited the correspondence between resonant and bound states of the inverted potential to obtain approximate black hole modes. WKB techniques were successfully used to calculate the first few least damped modes by Schutz and Will [8] and several subsequent authors 9, 10, 11, 12]. High precision QNMs for both Schwarzschild and Kerr black holes were first calculated by Leaver [13, 14] by means of a continued fraction approach.

\footnotetext{
* Presently performing national service with the Greek army.
} 
His results for the Schwarzschild case were later verified (and extended) by Andersson 15] via the phase-amplitude formalism and Nollert and Schmidt [16] who used a Laplace transform/analytic continuation method. Moreover, the semi-analytic phase-integral formalism [18] has proved to be a valuable tool for the calculation and interpretation of QNMs [19].

More recently, Onozawa [20] extended Leaver's calculation of the QNMs of rotating black holes and studied the behaviour of the mode frequencies as the rotation parameter is varied. The "long-lived" QNMs of a near extreme Kerr black hole were discussed by the present authors 21], using analytic expressions for the asymptotic amplitudes derived by Press and Teukolsky 22]. That study complemented a calculation by Detweiler who was the first to use these amplitudes to derive a QNM-condition for near extreme rotation, and moreover, solved it analytically for the extreme case [6]. Finally, Berti and Kokkotas [23] have used the continued fraction approach to study the behaviour of the high overtone QNMs of a Kerr black hole. Their study unveils a complicated, and quite puzzling, structure.

In the last few months there has been renewed interest in the spectrum of black hole QNMs. Motivated by the suggestion that the asymptotic behaviour of the high-overtone QNM frequencies 24, 25] may be related to the so-called Barbero-Immirzi parameter which is relevant for loop quantum gravity [26, 27], there has been several attempts to approximate the high-frequency part of the spectrum. Some progress has been achieved by linking the high-overtone spectrum with the black hole's quantum properties [28]. Although we will not discuss this issue further in the present article, it is an interesting problem that need to be investigated in complete detail.

The notion of Regge poles has (so far) hardly at all been discussed in the context of black holes. The Regge poles are close relatives to the QNMs [29], and correspond to those complex values of the angular momentum $l$ for which the wavefunction is purely outgoing at infinity and purely ingoing at the horizon. The Regge pole calculation poses an easier problem than that of finding QNMs since the corresponding wavefunction is well behaved at infinity (and the horizon) for complex $l$ (as long as the frequency is kept real). In quantum theory, the complex angular momentum (CAM) formalism (in which Regge poles play a central role) provides an alternative (and usually more transparent) description of scattering problems [30]. Some years ago, one of us applied the CAM theory to the study of scattering of massless scalar waves by a Schwarzschild black hole [31, 32]. In that study, Regge poles and the corresponding residues were calculated via the phase-integral method. The CAM theory has also been used by Chandrasekhar and Ferrari 33] in an investigation of axisymmetric pulsations of a relativistic star.

In this work we discuss numerical methods for calculating both QNMs and Regge poles. The key motivation for our study was the desire to devise methods that were easy to implement (hence "quick and dirty") and yet able to produce numerically accurate results. To achieve this goal we express the Teukolsky wave function in terms of suitable phase-functions (see Section III below). The integration of the resulting first-order equations readily yields the desired ratio of asymptotic amplitudes. In order to retain sufficient numerical precision to be able to identify the ingoing wave at infinity for complex frequencies, while ensuring that the phase-functions remain smooth and well behaved, the integration is generally performed along straight lines in the complex $r_{*}$ plane. The slope of the integration contour is chosen in such a way that the relative magnitude of the out- and ingoing wave solutions is preserved both near the horizon and at infinity. The end result is a multi-purpose scheme that (with minor modifications) facilitates the calculation of QNMs and Regge poles as well as scattering phase-shifts [34].

The remainder of the Paper is organized as follows: In Section II we briefly discuss the formal definition and physical interpretation of black hole resonances. Our numerical approaches to the problem are described in full detail in Section III. Section IV contains numerical results for QNMs (Section IVA) and Regge poles (Section IVB). Section V provides a summary and some final comments. Geometric units $G=c=1$ are adopted throughout the paper.

\section{BLACK HOLE RESONANCES: DEFINITION AND PHYSICAL MEANING}

The notion of scattering resonances is probably most familiar from quantum theory [35]. The fact that it is also relevant in black hole physics (in terms of QNMs and Regge poles) is not particularly surprising given that linear perturbations of black hole spacetimes are governed by Schrödinger type equations. The corresponding effective potential barriers are a combination of the black hole's gravitational pull and a repulsive centrifugal term [36]. This means that one can formulate a scattering problem for black holes and investigate phenomena analogous to those in the quantum case. For simplicity we will mainly consider the case of scalar waves in this paper. This is not a severe restriction, however, since more "realistic" cases, eg. gravitational perturbations, can be dealt with in exactly the same way.

After adopting the Boyer-Lindquist coordinate frame, and separating the angular dependence in the standard way, we arrive at the Teukolsky equation [37], which governs the radial component $\psi(r)$ of a scalar perturbation in a Kerr black hole spacetime,

$$
\frac{d^{2} \psi}{d r_{*}^{2}}+Q_{l m}\left(r_{*}, \omega, a\right) \psi=0
$$


We have also assumed a harmonic time dependence $e^{-i \omega t}$. The tortoise coordinate $r_{*}$ is defined as

$$
\frac{d r_{*}}{d r}=\frac{r^{2}+a^{2}}{\Delta}
$$

where $\Delta=\left(r-r_{+}\right)\left(r-r_{-}\right)$and $r_{ \pm}=M \pm\left(M^{2}-a^{2}\right)^{1 / 2}$ denote the black hole's inner (Cauchy) and outer (event) horizon. Explicitly we have

$$
r_{*}=r+\frac{2 M r_{+}}{r_{+}-r_{-}} \ln \left(\frac{r}{r_{+}}-1\right)-\frac{2 M r_{-}}{r_{+}-r_{-}} \ln \left(\frac{r}{r_{-}}-1\right)
$$

The effective potential $Q_{l m}$ is given by

$$
Q_{l m}\left(r_{*}, \omega, a\right)=\frac{K^{2}+\left(2 a m \omega-a^{2} \omega^{2}-E_{l m}\right) \Delta}{\left(r^{2}+a^{2}\right)^{2}}-\frac{d G}{d r_{*}}-G^{2}
$$

with $K=\left(r^{2}+a^{2}\right) \omega-a m$ and $G=r \Delta /\left(r^{2}+a^{2}\right)^{2}$. It has the following asymptotic behaviour

$$
Q_{l m} \sim \begin{cases}k^{2} & \text { as } r \rightarrow r_{+}\left(r_{*} \rightarrow-\infty\right), \\ \omega^{2} & \text { as } r \rightarrow+\infty\left(r_{*} \rightarrow \infty\right)\end{cases}
$$

where $k=\omega-m \omega_{+}$with $\omega_{+}=a / 2 M r_{+}$denoting the angular frequency of the event horizon. The eigenvalue of the relevant angular equation is denoted by $E_{l m}$, cf. [37].

As a result of (5), the solution of (10) will (to leading order) be a linear combination of simple exponentials as $r_{*} \rightarrow \pm \infty$. A physically acceptable solution describes a wave propagating towards the horizon at $r_{*} \rightarrow-\infty$, i.e. a purely "ingoing" solution. We can normalize this solution according to

$$
\psi^{i n} \sim\left\{\begin{array}{l}
e^{-i k r_{*}} \text { as } r_{*} \rightarrow-\infty \\
A_{\text {in }} e^{-i \omega r_{*}}+A_{\text {out }} e^{i \omega r_{*}}
\end{array} \text { as } r_{*} \rightarrow+\infty .\right.
$$

Another useful solution is purely "outgoing" at infinity,

$$
\psi^{u p} \sim e^{i \omega r_{*}}
$$

The Wronskian of any two linearly independent solutions of (10) is constant. Using the two solutions above we easily get

$$
W\left(\psi^{i n}, \psi^{u p}\right)=2 i \omega A_{\text {in }}
$$

Finally, we define the $\mathcal{S}$-matrix, a quantity familiar from scattering theory [35], as the ratio

$$
\mathcal{S}(\omega, l) \equiv(-1)^{\ell+1} \frac{A^{\text {out }}}{A^{\text {in }}}=\exp \left(2 i \delta_{l}\right)
$$

where $\delta_{l}$ defines the scattering phase-shifts.

One can define two classes of "resonances" that correspond to purely ingoing waves at the horizon and purely outgoing waves at infinity. Loosely speaking, such solutions are "characteristic" of the black hole spacetime since they do not depend on waves coming in from infinity. The first class of resonances are the QNMs. They correspond to complex frequency poles $\omega_{n}$ of $\mathcal{S}$ (for integer $l$ ). The QNM frequencies (obviously) correspond to solutions such that the Wronskian (8) vanishes. The second possible class of resonances are the Regge poles. To identify these one allows the angular momentum $(l)$ to assume complex values (while keeping $\omega$ real) and searches for poles $l_{n}$ of $\mathcal{S}$. The close connection between these classes of solutions has recently been discussed by Décanini, Folacci and Jensen 29].

It is well-known that the numerical determination of QNMs requires considerable care. Since we are discussing (stable) damped modes, the QNM frequencies will have negative imaginary parts. It follows then, that each QNM eigenfunction $e^{-i \omega_{n} t} \psi^{\text {in }}\left(\omega_{n}, r_{*}\right)$ will grow exponentially both towards infinity and at the horizon. Since one must be able to filter out the ingoing-wave solution at infinity in order to identify a QNM, the calculation requires exponential precision. It should be pointed out that this problem is not physical: It arises because we are viewing only a part of the overall problem, as is clearly shown in discussions of the initial-value problem for perturbed black holes [3, 16, 40]. Those studies reveal the true physical meaning of QNMs: They describe part of the late time behaviour of the perturbing field as observed at some fixed distance from the black hole [16]. They can be viewed as arising as a part of the field is temporarily "trapped" near the scattering center (the black hole) and leaks out to infinity. The same 
picture arises in the study of QNMs of much simpler systems such as static objects scattering waves in flat spacetime [38, 39].

The QNMs arise in scattering situations that can be thought of as "time dependent" (strictly speaking, the evolution and scattering of initial data with compact support). Alternatively, one might consider a time-independent scattering "experiment". Namely, the scattering of monochromatic plane waves (where the time dependence is trivially given by $\sim \exp (-i \omega t)$ for any given $\omega)$. In this case, the most important "observable" quantity is the differential cross section, which is a measure of the scatterer's size as seen by waves scattered into a certain solid angle [35]. The field at infinity consists of the incoming plane wave plus a scattered piece, and can be written

$$
\psi\left(r_{*} \rightarrow+\infty\right) \sim \psi_{\text {plane }}+f(\theta) \frac{e^{i \omega r_{*}}}{r}
$$

where we have assumed, for simplicity, axisymmetric scattering (for a treatment of non-axisymmetric scattering by rotating black holes, see 34]). The cross section can be expressed in terms of the (complex-valued) scattering amplitude $f(\theta)$,

$$
\frac{d \sigma}{d \Omega}=|f(\theta)|^{2}
$$

For scalar waves scattered off a Schwarzschild black hole one finds that [1],

$$
f(\theta)=\frac{1}{2 i \omega} \sum_{l=0}^{\infty}(2 l+1)\left(\mathcal{S}_{l}-1\right) P_{l}(\cos \theta)
$$

from which we see that all the relevant scattering information is encoded in the $\mathcal{S}$-matrix.

The angular scattering problem is usually studied in terms of the phase-shifts $\delta_{l}$. However, it is not unusual for the sum in (12) to be slowly converging (black-hole scattering being a example, although this difficulty can to some extent be avoided following the prescription in [42]). In many situations it is therefore desirable to have a different representation for the scattering amplitude. The most celebrated alternative is the CAM approach [30]. By means of a Watson-Sommerfeld transformation the sum over $l$ in (12) can be rewritten as an integral along a contour in the complex $l$-plane [31]. This integral naturally splits into two terms,

$$
f(\theta)=f_{P}(\theta)+f_{B}(\theta)
$$

The first term is a sum over the residues of the poles $l_{n}$ of $\mathcal{S}$; the Regge poles [35]. It is explicitly given by,

$$
f_{P}(\theta)=-\frac{i \pi}{\omega} \sum_{n=0}^{\infty} \frac{r_{n}\left(l_{n}+1 / 2\right)}{\cos \pi\left(l_{n}+1 / 2\right)} P_{l_{n}}(-\cos \theta)
$$

where $r_{n}$ is the residue of each Regge pole, i.e. is defined by

$$
\mathcal{S} \approx \frac{r_{n}}{l-l_{n}}
$$

in the vicinity of the $n^{\text {th }}$ pole, and $P_{l}$ is a Legendre function of complex degree. The second term in (13) is a "background integral", which is known to be less relevant as far as diffraction effects are concerned. As was demonstrated in [32], only the first few poles are required for determining the scattering cross section for angles away from the forward direction (the direction of incoming plane wave). Despite the fact that the problem of scattering by rotating black holes has not yet been studied within the CAM framework, it is natural to define Kerr black hole Regge poles as singularities of $\mathcal{S}$ in the complex $l$ plane while keeping $\omega$ real and $m$ an integer.

The physical interpretation of Regge states is discussed in standard textbooks [30, 35], cf. also the discussion of scattering of plane waves by a Schwarzschild black hole in [32]. By approximating the Legendre function for $\left|l_{n}+1 / 2\right| \gg 1$ and $\theta$ not too close to 0 or $\pi$ one can show that

$$
P_{l_{n}}(-\cos \theta) \approx\left[\frac{1}{2 \pi\left(l_{n}+1 / 2\right) \sin \theta}\right]^{1 / 2}\left\{e^{i\left(l_{n}+1 / 2\right)(\pi-\theta)-i \pi / 4}+e^{-i\left(l_{n}+1 / 2\right)(\pi-\theta)+i \pi / 4}\right\}
$$

From this formula it follows that each Regge pole can be interpreted as a combination of two "surface" waves traveling around the black hole in opposite directions. These waves decay exponentially as they propagate, with an "angular life" $\sim 1 / \operatorname{Im}\left(l_{n}+1 / 2\right)$. The real part of the Regge pole is associated with the distance $R_{n}$ from the black hole at which the angular decay occurs, $\operatorname{Re}\left(l_{n}+1 / 2\right) \approx \omega R_{n}$ (note that this "localization principle" is formally valid only for $l \gg 1$ [35]). As can be verified using the numerical results presented in Section VI, the value of $R_{n}$ lies very close to the critical impact parameter for null geodesics (which is $b_{\mathrm{c}}=3 \sqrt{3} M$ for a Schwarzschild black hole). This suggests that (as one would have expected) Regge states describe waves temporarily trapped in the vicinity of the unstable photon orbit, leaking to infinity and to the horizon, as they travel around the black hole. 


\section{TWO "QUICK AND DIRTY" SCHEMES}

In this Section we will discuss two closely related methods for determining black-hole resonances. Both methods are based on the idea of integrating slowly varying phase-functions rather than the, possibly rapidly, oscillating wavefunction itself. The representation of the solutions to Schrödinger type equations in terms of phase-functions was first discussed by Milne [43] and, a long time later, by Newman and Thorson [44]. The second key ingredient in our schemes is the use of complex values of the radial coordinate. By analytically continuing the resonant solutions into the complex coordinate plane one can suppress the divergences associated with (in particular) the QNM boundary conditions. In essence, our methods are close relatives to the phase-amplitude method that was developed by one of us [15] to study of scattering resonances. The main difference is that the methods we describe here make use of much simpler integration contours, and are consequently much easier to implement.

\section{A. The Prüfer method}

The family of the so-called Prüfer transformations has been widely used in the numerical analysis of Sturm-Liouville problems [45]. It has also been applied to the calculation of resonances and phase-shifts in standard quantum scattering problems, see for example Refs. [46, 47].

Consider a Schrödinger-type equation, where either the frequency $\omega$ or the angular momentum $l$ are allowed to take complex values,

$$
\frac{d^{2} \psi}{d x^{2}}+Q(x, l, \omega) \psi=0
$$

We consider cases where the radial coordinate $x$ spans the entire real axis (in the black hole case $x$ corresponds to the tortoise coordinate $r_{*}$ ), and the effective potential $Q$ is a single barrier with asymptotic behaviour

$$
Q \sim \begin{cases}q^{2} & \text { as } x \rightarrow-\infty \\ \omega^{2} & \text { as } x \rightarrow+\infty\end{cases}
$$

where $q=q(\omega)$. It follows then, that the function $\psi(x, l, \omega)$ will be a linear combination of exponentials as $x \rightarrow \pm \infty$. Following the discussion in the previous Section, let us assume that we are interested in a solution to (17) which is purely "ingoing" as $x \rightarrow-\infty$ and mixed ingoing/outgoing as $x \rightarrow+\infty$. That is, we want to determine the "in" solution, see eqn. (6), which can be written

$$
\psi \sim\left\{\begin{array}{l}
e^{-i q x} \text { as } x \rightarrow-\infty \\
B \sin [\omega x+\zeta] \text { as } x \rightarrow+\infty,
\end{array}\right.
$$

where $\zeta$ and $B$ are some complex constants.

We can write the exact solution of (17) in the form

$$
\psi(x, l, \omega)=\exp \left[\int P(x, l, \omega) d x\right]
$$

where $P$ is the logarithmic derivative of $\psi$ (a prime denotes derivative with respect to $x$ ),

$$
\frac{\psi^{\prime}}{\psi}=P
$$

This is our first phase-function. Obviously, it should obey the boundary condition $P \rightarrow-i q$ for $x \rightarrow-\infty$. Alternatively, we can express the function $\psi$ and its derivative via the transformation,

$$
\begin{aligned}
\psi(x, l, \omega) & =B \sin [\omega x+\tilde{P}(x, l, \omega)] \\
\psi^{\prime}(x, l, \omega) & =B \omega \cos [\omega x+\tilde{P}(x, l, \omega)]
\end{aligned}
$$

where $\tilde{P}$ is the Prüfer phase function (which should have $\zeta$ as its limiting value as $x \rightarrow+\infty$ ).

The two phase functions satisfy non-linear first order differential equations;

$$
\frac{d P}{d x}+P^{2}+Q=0
$$




$$
\frac{d \tilde{P}}{d x}+\left(\omega-\frac{Q}{\omega}\right) \sin ^{2}(\omega x+\tilde{P})=0
$$

and they are directly related by

$$
\tilde{P}=-\omega x+\frac{1}{2 i} \log \left[\frac{i P-\omega}{i P+\omega}\right]
$$

or

$$
P=\omega \cot (\omega x+\tilde{P})
$$

A major advantage of using phase-functions can be seen immediately from Eqns. (24) and (25), assuming for the moment that we are dealing with real $\omega$ and $l$. Using (18), we see that (24) and (25) lead to

$$
\begin{aligned}
& \frac{d P}{d x} \approx 0 \text { as } \quad x \rightarrow-\infty \\
& \frac{d \tilde{P}}{d x} \approx 0 \text { as } \quad x \rightarrow+\infty
\end{aligned}
$$

Hence, the functions $P$ and $\tilde{P}$ are slowly varying for large negative and positive values of $x$, respectively. This means that the oscillatory behaviour of the original wavefunction in those regimes [which would cause the build up of unnecessary numerical errors in a direct integration of [17)] has been effectively removed.

Finally, knowledge of the $\tilde{P}$ phase-function at $x \rightarrow+\infty$ provides us with the $\mathcal{S}$-matrix,

$$
\mathcal{S}(l, \omega)=(-1)^{l+1} e^{2 i \zeta}
$$

Obviously, the described method can be applied to the black-hole problem with $x \equiv r_{*}, q \equiv k$ and (17) becoming the Teukolsky radial equation (10).

At the practical level, the numerical integration for the $P$ function shoud be initiated at a point $r_{*}^{H}$ (say), close to the event horizon. Assuming that $Q$ is a slowly varying function for large $\left|r_{*}\right|$, which is certainly the case in the applications under consideration, we can initiate the integration of (24) from the lowest order WKB approximation

$$
P \approx \pm i Q^{1 / 2} \quad \text { as }\left|r_{*}\right| \rightarrow \infty
$$

(alternative approximations, eg. a power series expansion, will work equally well). In this way we can readily find a solution that corresponds to the (analytically continued) ingoing wave boundary condition at the horizon. This solution is then integrated towards the matching point $r_{*}^{\mathrm{ma}}$. As the integration reaches the vicinity of the potential peak (the local minimum of $Q$ in the Kerr case) we switch to the $\tilde{P}$ function, and then carry on the integration to "infinity". It is mandatory to change phase-function if one wants to ensure that the integrated function remains smooth and well behaved. This is due to the fact that the phase-functions undergo a drastic change in their behaviour (they become rapidly oscillating and may even have singularities) as they are extended through the region of the potential peak. This is a manifestation of the so-called Stokes phenomenon - the "switching on" of small exponentials in the solution to an equation of form (17), well known from WKB-and phase-integral theory [17, 18] (see also the discussion in Appendix 1 of [48]). This situation is demonstrated in Fig. 1 where we plot the functions $P\left(r_{*}\right)$ and $\tilde{P}\left(r_{*}\right)$ for typical parameters $l=m=2, \omega M=0.5, a=0.5 M$.

The Prüfer approach has already been applied in the context of scattering of plane scalar waves by rotating black holes [34]. In that study, where both $\omega$ and $l$ were assumed to be real, the use of the phase-functions described above facilitated the computation of accurate high frequency phase-shifts (which would be problematic if the original wave equation were to be integrated [42]). We can follow exactly the same procedure when $l$ is allowed to acquire complex values (as long as $\omega$ remains real). In this way we have calculated the first few Regge poles of both Schwarzschild and Kerr black holes. The numerical results will be discussed in the following Section.

Complications arise when $\omega$ becomes complex while $l$ is kept real (integer). From Eqn. (25) we see that the term which represents an ingoing (outgoing) wave becomes exponentially small (large) at large $x$ (for $\operatorname{Im} \omega<0$ ). A similar situation appears close to the horizon. Therefore, we are still facing one of the problems associated with a direct integration of the wave equation. An efficient and elegant way to avoid this difficulty is to consider the problem in the complex $r_{*}$-plane. The success of this approach has been repeatedly demonstrated with the application of the phase-integral/amplitude formalisms to the calculation of black-hole QNMs [15, 19, 55]. The main idea is to integrate our equations not along the real axis, but instead use a suitably chosen contour $C$ such that the ingoing/outgoing 

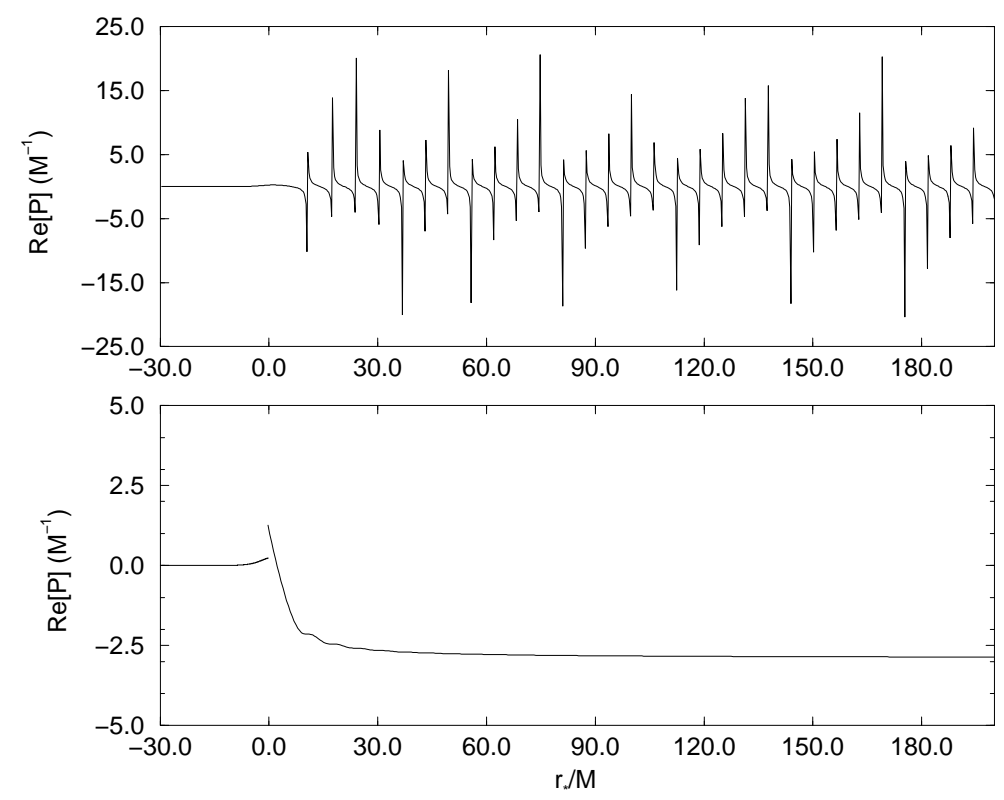

FIG. 1: The Prüfer phase-functions (only real parts shown) resulting from a typical integration of eqns (24) and 25) with parameters $l=m=2, \omega M=0.5, a=0.5 M$. Top panel: the integration of the $P$ function was continued beyond the potential peak (located close to $r_{*}=0$ ). Beyond this point its behaviour changes dramatically as a result of the Stokes phenomenon. As is clear from the graph, the derivative of $P$ takes particularly large values at a series of points beyond $r_{*}=0$. This may signal the existence of poles of the phase function close to the real $r_{*}$ axis. That this behaviour could be expected, if one fails to account for the Stokes phenomenon, is clear from the equations given in Appendix 1 of [48]. Bottom panel: as soon as the peak region is reached, we shift to the $\tilde{P}$ function (the matching is performed at the point where the curve "breaks" in the figure) which is much better behaved, basically because it describes both ingoing and outgoing waves, see eqn. (22). Tests show that the result does not depend on the choice of matching point, as long as it remains close to the peak of the effective black-hole potential.

wave solutions, eg. $e^{ \pm i \omega r_{*}}$, have comparable asymptotic magnitudes (of order unity) as $r_{*} \rightarrow \infty$. The simplest choice of integration contour is a straight line with slope $-\operatorname{Im} \omega / \operatorname{Re} \omega$, see Fig 2 Similarly, a line with slope $-\operatorname{Im} k / \operatorname{Re}$ $k$ will guarantee purely oscillatory behaviour near the event horizon. Such paths are parallel (for $\left|r_{*}\right| \rightarrow \infty$ ) to the so-called anti-Stokes lines which play a crucial role in WKB and phase-integral theory [15, 18]. The anti-Stokes lines are curves along which $\int Q^{1 / 2} d r_{*}$ is purely real and as a consequence the WKB solution of (11) is purely oscillatory. It should be noted that a similar integration contour has been used in a calculation of relativistic stellar pulsation modes [49].

We use a parameterization

$$
r_{*}=r_{*}^{\mathrm{ma}}+\rho e^{i \beta}
$$

with $-\infty<\rho<+\infty$ and $\beta$ given by,

$$
\beta=\left\{\begin{array}{l}
-\operatorname{Arg}(\omega) \quad \text { for } \rho>0 \\
-\operatorname{Arg}(k) \quad \text { for } \rho<0
\end{array}\right.
$$

The matching point $r_{*}^{\mathrm{ma}}$ is chosen to lie on the real axis $(\rho=0)$ and some experimentation shows that reliable results are obtained as long as the matching is performed near the peak of the curvature potential (the extremum of $Q$ ).

Rewriting Eqns. (24) and (25) in terms of $\rho$ we have

$$
\begin{gathered}
\frac{d P}{d \rho}+e^{-i \beta}\left(P^{2}+Q\right)=0 \\
\frac{d \tilde{P}}{d \rho}+e^{-i \beta}\left(\omega-\frac{Q}{\omega}\right) \sin ^{2}\left(\tilde{P}+|\omega| \rho+\omega r_{*}^{\text {ma }}\right)=0
\end{gathered}
$$

The radial coordinate $r$ will also be assumed complex, and we determine it from

$$
\frac{d r}{d \rho}=e^{-i \beta} \frac{\Delta(r)}{r^{2}+a^{2}}
$$




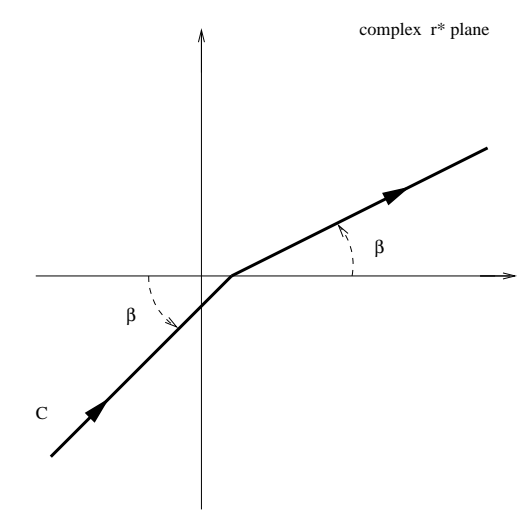

FIG. 2: The integration path $C$ in the complex $r_{*}$-plane used to calculate QNMs. The slope $\beta$ is chosen in such a way that divergent/decaying solutions at infinity and at the horizon are avoided.

With the help of (3) it is easy to see that

$$
r \approx r_{+}\left(1+e^{c r_{*}}\right)
$$

where $c=\left(r_{+}-r_{-}\right) / 2 M r_{+}$as $\rho \rightarrow-\infty$, and that $r \approx r_{*}$ as $\rho \rightarrow+\infty$. Hence, the proposed integration contour corresponds to a straight line at large distances in the $r$-plane, and to a clockwise spiral as the horizon is approached. This strongly resembles the more complicated integration contour used in the phase-amplitude calculation of QNMs, see figures 1 and 2 in $[15]$.

Not surprisingly, some care must be taken in implementing complex integration contours in black-hole problems. For example, we need to understand the analytic properties of the potential $Q$. From Eqn. (4) we deduce that this quantity will have poles at $r= \pm i a$. Since the function $r_{*}(r)$ is multivalued, there will be a series of poles in the left half of the $r_{*}$-plane, symmetrically located with respect to the real axis. All of these poles have the same real part (which is zero for the non-rotating case), while their imaginary parts satisfy $\operatorname{Im} r_{*} \gg \operatorname{Re} r_{*}$. In practice, the presence of these singularities provides an upper limit to the complex-rotation angle. This is easiest illustrated by the Schwarzschild case. According to (3) the point $r=0$ translates into

$$
r_{*}(0)=2 M \log (-1)= \pm 2 M i \pi(1+2 k) \quad \text { with } \quad k=0,1,2, \ldots
$$

It is easy to understand that one should not rotate the integration contour beyond this angle.

Another important issue that deserves some discussion concerns the boundary conditions in the complex plane. When we are solving the system of phase equations (34) and (35) we are actually imposing the QNM boundary condition on the contour $C$ at $\rho \rightarrow \pm \infty$. However, the "true" boundary conditions are to be imposed on the real axis $\left(r_{*} \rightarrow \pm \infty\right)$. It is perfectly natural to wonder if we are, in some way, altering the problem by using the contour $C$. This question has been discussed in the context of WKB and phase-integral studies (and the complex $r$-plane) [18, 50]. It was concluded that it is safe to analytically continue the boundary condition in the proposed way.

\section{B. QNM excitation coefficients}

We have used the Prüfer transformation scheme described above to calculate both QNMs and Regge poles for scalar waves in the geometry of a rotating black hole. The method is easily extended to other classes of perturbing fields, the most interesting of which is likely to be that of gravitational perturbations. As will be discussed below the method is somewhat limited for extremely rapidly damped QNMs, and hence it cannot be used to investigate the asymptotic behaviour of the mode spectrum 27]. However, from an astrophysical point of view the slowly damped modes are the most important and for these our scheme works very well, indeed.

In the case of QNMs one might also want to assess to what extent the modes are excited given a certain initial perturbation. As discussed in [40] the answer can be quantified in an initial-data independent way in terms of the "excitation coefficient"

$$
B_{n}=\frac{A_{\text {out }}\left(\omega_{n}\right)}{2 \omega_{n} \alpha_{n}}
$$

where we have assumed that $A_{\text {in }} \approx \alpha_{n}\left(\omega-\omega_{n}\right)$ in the vicinity of the $n^{\text {th }}$ mode frequency. While one can formulate expressions for this quantity within our previous scheme, we have found that the precision of the actual numerical 
results is somewhat unsatisfactory. To remedy this we have devised an alternative scheme. The purpose of the following discussion is to present this scheme, with the particular aim of achieving accurate results for the excitation coefficients.

The first half of the calculation remains identical to the one discussed above, and we thus obtain a solution $P_{L}\left(r_{*}^{\mathrm{ma}}\right)$ (say) which satisfies that boundary condition of ingoing waves at the event horizon. At spatial infinity we now obtain the appropriate solution in a similar way. Let us choose the phase of the square root in such a way that the solution corresponding to $P_{+} \approx+i Q^{1 / 2}\left(r_{*}\right)$ has the desired outgoing-wave character. Once this solution is extended to the matching point we have a second solution $P_{+}\left(r_{*}^{\mathrm{ma}}\right)$. Thus a resonant overall solution, a QNM, follows from the simple condition

$$
P_{+}\left(r_{*}^{\mathrm{ma}}\right)=P_{L}\left(r_{*}^{\mathrm{ma}}\right)
$$

Now we want to determine also the excitation coefficients. We can do this by considering the solution for a general frequency, that contains both in- and outgoing wave at infinity, rather than a specific QNM solution. Assume (as before) that $P_{+} \approx+i Q^{1 / 2}\left(r_{*}\right)$ corresponds to an outgoing wave solution, and that $P_{-} \approx-i Q^{1 / 2}\left(r_{*}\right)$ represents an ingoing wave. This means that, after integrating these two functions to the matching point the general solution corresponds to the linear combination

$$
\psi_{R}\left(r_{*}^{\mathrm{ma}}\right)=A \exp \left[\int_{r_{*}^{\infty}}^{r_{*}^{\mathrm{ma}}} P_{+} d r_{*}\right]+B \exp \left[\int_{r_{*}^{\infty}}^{r_{*}^{\mathrm{ma}}} P_{-} d r_{*}\right]
$$

where the integration is initiated at $r_{*}^{\infty}$. We can write the logarithmic derivative of this function as

$$
\frac{\psi_{R}^{\prime}}{\psi_{R}}=\frac{P_{+}+(B / A) P_{-} \exp \left[\int_{r_{*}^{\infty}}^{r_{*}^{\mathrm{ma}}}\left(P_{-}-P_{+}\right) d r_{*}\right]}{1+(B / A) \exp \left[\int_{r_{*}^{\infty}}^{r_{*}^{\text {ma }}}\left(P_{-}-P_{+}\right) d r_{*}\right]}
$$

The condition for an overall solution at $r_{*}^{\text {ma }}$ is that the logarithmic derivative is continuous, i.e. that the above expression is equal to $P_{L}\left(r_{*}^{\mathrm{ma}}\right)$. This condition can be written

$$
\frac{B}{A}=\frac{P_{+}-P_{L}}{P_{L}-P_{-}} \exp \left[\int_{r_{*}^{\infty}}^{r_{*}^{\mathrm{ma}}}\left(P_{+}-P_{-}\right) d r_{*}\right] \quad \text { for } r_{*}=r_{*}^{\mathrm{ma}}
$$

Now recalling that $P_{-}$corresponds to the ingoing wave solution at infinity we know that a QNM must correspond to $B=0$, and we see that the above condition is the same as the one we derived previously.

To determine the excitation coefficient requires a little more work. First we need to relate our amplitudes $A$ and $B$ to the asymptotic amplitudes $A_{\text {out }}$ and $A_{\text {in }}$. Recall that the asymptotic amplitudes are determined from

$$
\psi \sim A_{\mathrm{out}} e^{i \omega r_{*}}+A_{\mathrm{in}} e^{-i \omega r_{*}} \quad \text { as } r_{*} \rightarrow \infty
$$

We want to compare this expression to our solutions. Since we have assumed that the WKB approximation is valid at the point where we initiate integration $\left(r_{*}^{\infty}\right)$ we can use

$$
\int_{r_{*}^{\infty}}^{r_{*}} P_{ \pm} d r_{*} \approx \pm i \int_{r_{*}^{\infty}}^{r_{*}} Q^{1 / 2} d r_{*}= \pm i \omega\left(r_{*}-r_{*}^{\infty}\right) \pm i \int_{r_{*}^{\infty}}^{r_{*}}\left(\frac{Q-\omega^{2}}{Q^{1 / 2}+\omega}\right) d r_{*}
$$

beyond $r_{*}^{\infty}$ (the last integral should be easy to evaluate). We then have

$$
\psi_{+} \approx A \exp \left[\int_{r_{*}^{\infty}}^{r_{*}} P_{+} d r_{*}\right]=A \exp \left[i \omega\left(r_{*}-r_{*}^{\infty}\right)+i \int_{r_{*}^{\infty}}^{r_{*}}\left(\frac{Q-\omega^{2}}{Q^{1 / 2}+\omega}\right) d r_{*}\right] \text { for }\left|r_{*}\right|>\left|r_{*}^{\infty}\right|
$$

And we can identify

$$
A_{\mathrm{out}}=A \exp \left[-i \omega r_{*}^{\infty}+i \int_{r_{*}^{\infty}}^{\infty}\left(\frac{Q-\omega^{2}}{Q^{1 / 2}+\omega}\right) d r_{*}\right]
$$

Similarly, we get

$$
A_{\text {in }}=B \exp \left[i \omega r_{*}^{\infty}-i \int_{r_{*}^{\infty}}^{\infty}\left(\frac{Q-\omega^{2}}{Q^{1 / 2}+\omega}\right) d r_{*}\right]
$$


Collecting the above results, we have

$$
\frac{A_{\text {in }}}{A_{\text {out }}}=\frac{P_{+}-P_{L}}{P_{L}-P_{-}} \exp \left[\int_{r_{*}^{\infty}}^{r_{*}^{\mathrm{ma}}}\left(P_{+}-P_{-}\right) d r_{*}+2 i \omega r_{*}^{\infty}-2 i \int_{r_{*}^{\infty}}^{\infty}\left(\frac{Q-\omega^{2}}{Q^{1 / 2}+\omega}\right) d r_{*}\right]
$$

The final step in the derivation consists of taking the derivative of this expression with respect to $\omega$ and evaluating it at a QNM frequency. This immediately leads to

$$
\frac{\alpha_{n}}{A_{\mathrm{out}}}=\frac{1}{P_{L}-P_{-}}\left[\frac{d}{d \omega}\left(P_{+}-P_{L}\right)\right] \exp \left[\int_{r_{*}^{\infty}}^{r_{*}^{\mathrm{ma}}}\left(P_{+}-P_{-}\right) d r_{*}+2 i \omega r_{*}^{\infty}-2 i \int_{r_{*}^{\infty}}^{\infty}\left(\frac{Q-\omega^{2}}{Q^{1 / 2}+\omega}\right) d r_{*}\right]
$$

from which we can calculate the desired excitation coefficient.

To conclude, let us summarize the additional steps that are required in a calculation of the excitation coefficient

once a QNM frequency has been determined. First we need to evaluate the two integrals $\int_{r_{*}^{\infty}}^{r_{*}^{\mathrm{ma}}} P_{ \pm} d r_{*}$. This is best done by introducing additional dependent variables, let us call them $\Phi_{ \pm}$, and then integrating the equations

$$
\Phi_{ \pm}^{\prime}=P_{ \pm}
$$

together with (24) from $r_{*}^{\infty}$ to $r_{*}^{\mathrm{ma}}$. Secondly, we need the derivatives $\frac{d P_{+}}{d \omega}$ and $\frac{d P_{L}}{d \omega}$. These quantities are also best calculated by introducing additional variables, i.e. by integrating

$$
\frac{d}{d r_{*}}\left(\frac{d P_{ \pm}}{d \omega}\right)=-\frac{d Q}{d \omega}-2 P_{ \pm} \frac{d P_{ \pm}}{d \omega}
$$

using appropriate initial data, i.e.

$$
\frac{d P_{ \pm}}{d \omega} \approx \pm \frac{i}{2 Q^{1 / 2}} \frac{d Q}{d \omega}
$$

at $r_{*}^{\infty}$. Thirdly, we need to evaluate

$$
\int_{r_{*}^{\infty}}^{\infty}\left(\frac{Q-\omega^{2}}{Q^{1 / 2}+\omega}\right) d r_{*}
$$

This is readily done by first expanding the integrand in inverse powers of $r$ and then integrating. The result is convergent in all relevant cases.

\section{Numerical implementation}

Before concluding the description of the methods we have devised to study black hole resonances, it is worth commenting on the numerical implementation of the schemes. Overall we have a system of two coupled nonlinear first-order equations to solve numerically (for either choice of phase-function), namely, eqns (34) or (35) together with (36). In reality, since we are dealing with intrinsically complex quantities, we split each equation in its real and imaginary parts. At the end of the day, we arrive at four equations to be solved. This is done by means of a standard variable-step Addams method.

To complete the solution of the problem, we combine the result of the integration of the phase functions with a complex root-finder routine (making use of Müller's algorithm [51]) to search the complex frequency/angular momentum plane for zeros of the ratio $A_{\text {in }} / A_{\text {out }}$. A prior knowledge of the approximate location of a resonance greatly speeds up the overall procedure.

\section{NUMERICAL RESULTS}

The main aim of this investigation was to devise simple and efficient methods for computing black-hole resonances and scattering phase-shifts. As should be clear from the previous Sections, the phase-function schemes that we are discussing are straightforward to implement. They also turn out to be numerically robust and provide results of excellent accuracy. We have tested the methods by calculating known results for both Schwarzschild and Kerr 


\begin{tabular}{|r|r|r|r|}
\hline$l$ & $n$ & $\omega_{n} M$ & \multicolumn{1}{c|}{$B_{n}$} \\
\hline 2 & 0 & $0.373672-0.088962 i$ & $0.126845+0.020669 i$ \\
& 1 & $0.346711-0.273915 i$ & $0.048328-0.223618 i$ \\
& 2 & $0.301052-0.478276 i$ & $-0.190336+0.015118 i$ \\
& 3 & $0.251504-0.705148 i$ & $0.080519+0.079964 i$ \\
& 4 & $0.207514-0.946845 i$ & $-0.016727-0.060630 i$ \\
& 5 & $0.169299-1.195608 i$ & $-0.001566+0.035783 i$ \\
\hline 3 & 0 & $0.599443-0.092703 i$ & $-0.093672-0.049606 i$ \\
& 1 & $0.582643-0.281298 i$ & $-0.152350+0.269067 i$ \\
& 2 & $0.551683-0.479093 i$ & $0.414353+0.143038 i$ \\
& 3 & $0.511956-0.690338 i$ & $-0.041146-0.412997 i$ \\
& 4 & $0.470173-0.915660 i$ & $-0.220524+0.225274 i$ \\
\hline 4 & 0 & $0.809178-0.094164 i$ & $0.064924+0.065661 i$ \\
& 1 & $0.796631-0.284334 i$ & $0.263139-0.249790 i$ \\
& 2 & $0.772710-0.479904 i$ & $-0.546231-0.439093 i$ \\
& 3 & $0.739835-0.683916 i$ & $-0.323148+0.835511 i$ \\
& 4 & $0.701524-0.898240 i$ & $0.867222-0.114054 i$ \\
\hline
\end{tabular}

TABLE I: Numerical results for the first few QNMs, and the associated excitation coefficients $B_{n}$, corresponding to gravitational perturbations of a Schwarzschild black hole.

black holes. The obtained results serve as a validation of our numerical code(s). Having confirmed the reliability of our schemes we have extended the study to, in particular, the resonances of rotating black holes. Below, we will describe new results for both QNMs and Regge poles of Kerr black holes. These results shed interesting light on issues concerning the excitation of the long lived QNMs that are known to exist for near extreme Kerr black holes, the relation between the approximate QNMs for $a \rightarrow M$ used in (for example) [21], and also the effect of rotation on the black hole Regge poles.

\section{A. Quasinormal modes}

As a first application of our numerical methods, we have calculated the first QNMs of a Schwarzschild black hole. The obtained results agree perfectly with results in the literature 15], and confirm that our method is reliable as long as the modes are not damped too rapidly. The method breaks down when the imaginary part of the mode frequency is about one order of magnitude larger than the real part. This means that one should not expect to be able to use our scheme to study the asymptotic behaviour of the QNM spectrum. However, all astrophysically relevant QNMs are readily calculated. We have also tested the obtained excitation coefficients by comparing results obtained for gravitational perturbations (using a code based on the Sasaki-Nakamura equation [52]) to the phase-integral results in 53] and the data obtained by Leaver 13. A sample of results is given in Table 1 By comparing the listed results to those in Table II of [53] we conclude that the present results, which agree very well with those of Leaver apart from a few sign discrepancies already pointed out in [53], are reliable. In fact, we believe that the present method provides the (so far) most efficient tool for investigating the QNM excitation problem.

As the black hole acquires rotation, each of the Schwarzschild QNMs (for a given $l$ ) splits into $2 l+1$ distinct modes. We have tested the reliability of our scheme by reproducing the Kerr QNM frequencies obtained by Leaver, cf. Tables 2 and 3 in 13]. A sample of results for $l=2$, obtained by implementing our Prüfer phase function scheme for the Sasaki-Nakamura equation, are provided in Table IVA These results are in perfect agreement with those obtained by Leaver's continued fraction calculation for $m=0$ and $m= \pm 1$. Furthermore, Table IVA is useful since the $l=2$ QNMs may contribute significantly to the gravitational-wave signal from, for example, a black hole born following a supernova core collapse or a compact binary merger (see [54 for discussion).

Kerr QNMs are known to exhibit interesting behaviour as a varies [6]. In particular, we see from Table IVA that rotation has a pronounced effect on modes with $m=l>0$. These can be thought of as "equatorial" and "co-rotating" with the black hole (cf. the symmetry properties of the associated angular functions, which in the case of scalar waves are spheroidal harmonics $\left.S_{l m}^{a \omega}(\theta)\right)$. In the limit $a \rightarrow M$ the co-rotating modes have been found to become long-lived $\left(\operatorname{Im} \omega_{n} M \rightarrow 0\right)$ and accumulate at the frequency $m \omega_{+}[6,14,20,21]$. Hence, rotation has a significant effect on these modes. In contrast, the "counter-rotating" modes $(m<0)$ are much less affected by rotation and remain relatively 


\begin{tabular}{|c|c|c|c|c|c|}
\hline$a / M$ & $m=2$ & $m=1$ & $m=0$ & $m=-1$ & $m=-2$ \\
\hline 0 & $0.373672-0.088962 i$ & $0.373672-0.088962 i$ & $0.373672-0.088962 i$ & $0.373672-0.088962 i$ & $0.373672-0.088962 i$ \\
0.10 & $0.387018-0.088706 i$ & $0.380432-0.088798 i$ & $0.374032-0.088898 i$ & $0.367812-0.089004 i$ & $0.361768-0.089114 i$ \\
0.20 & $0.402145-0.088311 i$ & $0.388248-0.088488 i$ & $0.375124-0.088700 i$ & $0.362738-0.088935 i$ & $0.351053-0.089182 i$ \\
0.30 & $0.419527-0.087729 i$ & $0.397330-0.087995 i$ & $0.376985-0.088353 i$ & $0.358366-0.088763 i$ & $0.341333-0.089184 i$ \\
0.40 & $0.439842-0.086882 i$ & $0.407979-0.087257 i$ & $0.379682-0.087827 i$ & $0.354633-0.088484 i$ & $0.332458-0.089131 i$ \\
0.50 & $0.464123-0.085639 i$ & $0.420632-0.086173 i$ & $0.383318-0.087069 i$ & $0.351491-0.088091 i$ & $0.324307-0.089031 i$ \\
0.60 & $0.494045-0.083766 i$ & $0.435969-0.084564 i$ & $0.388054-0.085995 i$ & $0.348911-0.087566 i$ & $0.316784-0.088892 i$ \\
0.70 & $0.532599-0.080794 i$ & $0.455122-0.082085 i$ & $0.394129-0.084453 i$ & $0.346870-0.086884 i$ & $0.309808-0.088717 i$ \\
0.80 & $0.586015-0.075626 i$ & $0.480231-0.077955 i$ & $0.401917-0.082156 i$ & $0.345356-0.086003 i$ & $0.303313-0.088512 i$ \\
0.90 & $0.671634-0.064863 i$ & $0.516292-0.069804 i$ & $0.412004-0.078483 i$ & $0.344359-0.084865 i$ & $0.297244-0.088281 i$ \\
0.99 & $0.870861-0.029510 i$ & $0.572720-0.046209 i$ & $0.423685-0.072701 i$ & $0.343890-0.083550 i$ & $0.292107-0.088052 i$ \\
\hline
\end{tabular}

TABLE II: QNM frequencies $\left(\omega_{n} M\right)$ for gravitational perturbations of Kerr black holes with various spin rates. The data is for $l=2$, and illustrates that the co-rotating modes $m>0$ become long lived as the black hole approaches extreme rotation. In contrast the counter-rotating modes $m<0$ are much less affected by the rotation.

close to the initial Schwarzschild QNMs for all values of $a$. In Fig. 3 we illustrate this behaviour by showing the change in the first few scalar field QNM frequencies as the rotation rate of the black hole is varied. The data in the figure corresponds to $0 \leq a / M \leq 0.99$.

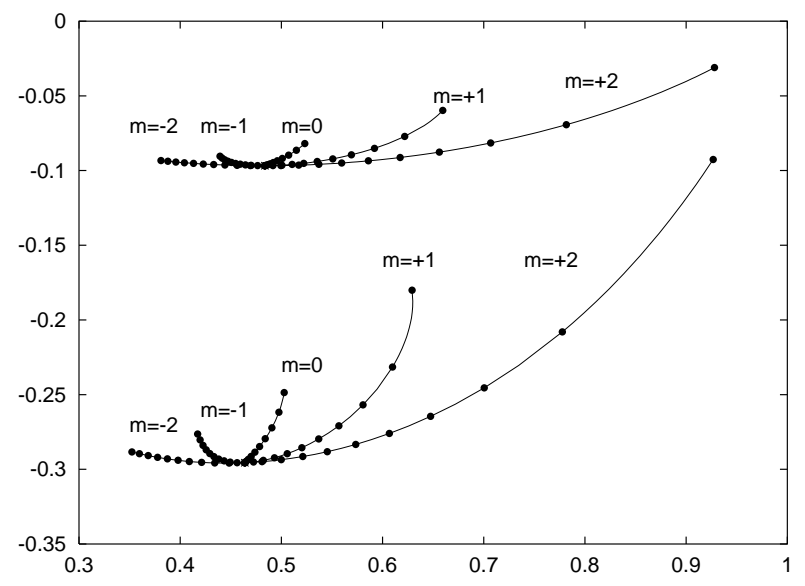

FIG. 3: The trajectory (Im $\omega M$ as a function of Re $\omega M$ ) of the first two $l=2$ scalar field Kerr QNMs for varying black hole rotation $0 \leq a / M \leq 0.99$. The position of each mode at spin values $a / M=0,0.1,0.2 \ldots 0.9,0.99$ is represented by dots.

The behaviour of the QNMs as the black hole approaches extreme rotation is particularly intriguing. Unfortunately, our method breaks down beyond $a \approx 0.999 \mathrm{M}$. The likely reason for this is the multivaluedness of the complex $r_{*}$ coordinate, cf. for example (38), and the need to initiate the integration close to the horizon, see eqn (37). This becomes tricky as the event horizon and the inner Cauchy horizon come close together. A similar problem is known to arise in the phase-integral study of the QNMs of a Reissner-Nordström black hole [55]. This means that we cannot push our calculation all the way to the extreme limit. Of course, we can determine QNMs for all rotation rates that are likely to be astrophysically relevant. Still, the behaviour in the extreme limit is interesting from a conceptual point of view. Especially since one can approximate the QNM spectrum for near extreme black holes $[\underline{6}$. In figure 4 we compare our numerical results for scalar field QNMs to approximate results for $a \approx M$ and $\omega \approx m \omega_{+}$. The figure shows that the numerical results for the first two $l=m=2$ QNMs, as extended from the Schwarzschild limit, approach the first two approximate modes as $a \rightarrow M$. This is very encouraging since it demonstrates a correspondence between two rather different computational schemes. It also provides support for any argument concerning the nature and relevance of these long-lived modes that is based on the approximate formulae. The comparison also indicates the range of validity of Detweiler's approximate mode-condition [6] in a useful way.

It has been argued that the existence of long-lived QNMs for rapidly rotating black holes could prove important for gravitational-wave astronomy. The basic idea is that a slowly damped mode will be easier to detect provided 


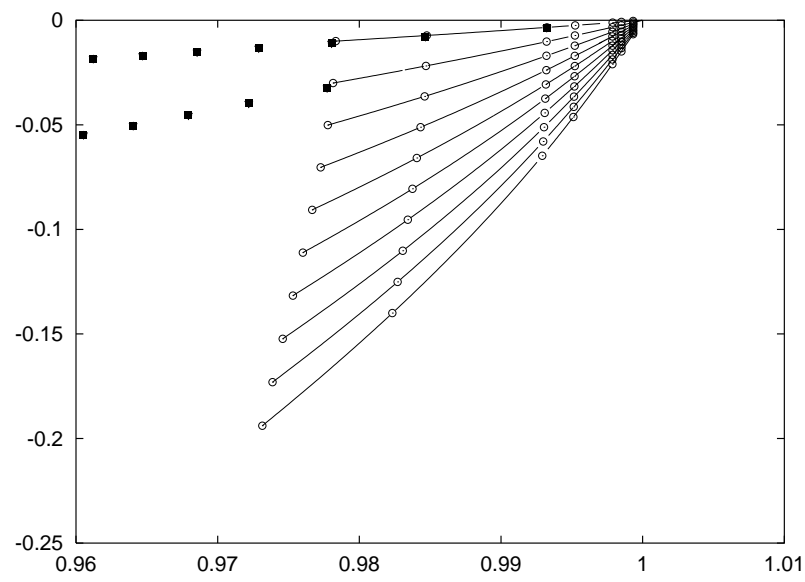

FIG. 4: Comparison of the numerical $l=m=2$ long-lived modes (the first two are shown here, denoted by boxes) with modes derived by solving Detweiler's approximate mode-condition, (represented by lines, with the circles denoting the modes at $a / M=0.999,0.9995, \ldots, 0.999999)$. It is clear that, for the fundamental mode, the two calculations are in agreement. The same seems to be true for the first overtone, although we have not been able to proceed numerically beyond $a=0.999 M$ in that case. The situation for the higher overtones also remain unclear.

\begin{tabular}{|c|cc|cc|}
\hline$a / M$ & \multicolumn{2}{|c|}{$m=+2$} & \multicolumn{2}{c|}{$m=-2$} \\
\hline & $\omega_{n} M$ & $B_{n}$ & $\omega_{n} M$ & $B_{n}$ \\
\hline 0.0 & $0.483644-0.096759 i$ & $0.5252+0.0950 i$ & $0.483644-0.096759 i$ & $0.5252+0.0950 i$ \\
0.1 & $0.499482-0.096666 i$ & $0.6708+0.2710 i$ & $0.469295-0.096708 i$ & $0.4354+0.0358 i$ \\
0.2 & $0.517121-0.096382 i$ & $0.8355+0.8432 i$ & $0.456198-0.096546 i$ & $0.3790+0.0132 i$ \\
0.3 & $0.536979-0.095839 i$ & $0.8370+1.8608 i$ & $0.444168-0.096299 i$ & $0.3412+0.0044 i$ \\
0.4 & $0.559647-0.094931 i$ & $-0.9646-0.0827 i$ & $0.433059-0.095984 i$ & $0.3145+0.0016 i$ \\
0.5 & $0.585990-0.093494 i$ & $-0.3913-0.2772 i$ & $0.422751-0.095616 i$ & $0.2950+0.0018 i$ \\
0.6 & $0.617364-0.091245 i$ & $-0.1359-0.2436 i$ & $0.413146-0.095204 i$ & $0.2803+0.0034 i$ \\
0.7 & $0.656099-0.087650 i$ & $0.0005-0.1699 i$ & $0.404163-0.094759 i$ & $0.2691+0.0057 i$ \\
0.8 & $0.706823-0.081520 i$ & $0.0638-0.0754 i$ & $0.395734-0.094285 i$ & $0.2607+0.0083 i$ \\
0.9 & $0.781638-0.069289 i$ & $0.0432+0.0143 i$ & $0.387799-0.093790 i$ & $0.2550+0.0108 i$ \\
0.99 & $0.928026-0.031069 i$ & $-0.0027-0.0030 i$ & $0.381041-0.093330 i$ & $0.2545+0.0123 i$ \\
\hline
\end{tabular}

TABLE III: QNM frequencies $\left(\omega_{n} M\right)$ and associated excitation coefficients $\left(B_{n}\right)$ for scalar perturbations of Kerr black holes with various spin rates. The data is for $l=2$ and $m= \pm 2$.

that it is initially excited to the same amplitude as a more rapidly damped mode. Since we now have the means to calculate the excitation coefficients for the Kerr QNMs we can analyse this problem quantitatively. As we have indicated in a previous paper [21], our results do not support the idea that the long-lived QNMs will be of particular importance. The reason for this can be seen from Table III], where we provide results for the fundamental $m= \pm 2$ QNMs for various values of the rotation parameter. From this data we see that while the absolute value of the excitation coefficient for the counter-rotating QNM only changes by about a factor of two as the black hole spins up towards the extreme limit, it drops by about two order of magnitude for the co-rotating mode. This indicates that the co-rotating long-lived QNMs may be significantly more difficult to excite in a dynamical process.

We can use the results in Table III to discuss the "detectability" of the long-lived QNMs. Following [21] we express the effective gravitational-wave amplitude associated with a single QNM (with complex frequency $\omega_{n}$ ) as

$$
h_{\mathrm{eff}} \propto \sqrt{\frac{\operatorname{Re} \omega_{n}}{\operatorname{Im} \omega_{n}}} \frac{A^{\text {out }}}{\alpha_{n}}=2 \sqrt{\frac{\operatorname{Re} \omega_{n}}{\operatorname{Im} \omega_{n}}} \omega_{n} B_{n}
$$

Given our numerical data, we can compare the result for co- and counter-rotating (scalar field) modes. Figure 5 (which is identical to Figure 1 in 21]) shows the outcome of this comparison. From the figure it is clear that, even though the co-rotating mode is much longer lived, its "detectability" decreases significantly as $a \rightarrow M$. We conclude that the present results suggest that the effective amplitude of the slowest damped QNM for a rapidly rotating black 
hole may be several orders of magnitude smaller than the amplitude of the corresponding mode of a Schwarzschild black hole, or indeed the counter-rotating Kerr modes. The possible constructive interference of the many long-lived QNMs that exist for near extreme Kerr black holes, and which could still lead to a relevant gravitational-wave signal, has been discussed in [21].

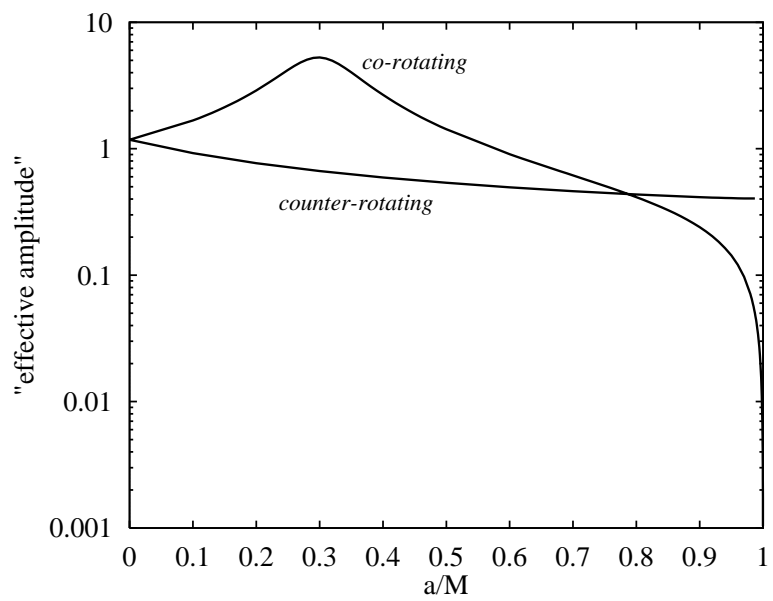

FIG. 5: Assessment of the "detectability" of Kerr black hole QNMs as $a \rightarrow M$. The effective amplitude $h_{\mathrm{eff}}$ (as estimated from Eq. (55) ) is plotted as a function of the spin parameter $a / M$. We compare the slowest damped co-rotating QNM to the slowest damped counter-rotating one. The figure shows that the co-rotating mode (which becomes very long lived in the near extreme case) has a much smaller excitation coefficient than the counter-rotating mode as $a \rightarrow M$.

As a final application of our method we have traced the path of a high overtone mode $(n=9, l=2, m=0)$ for varying black-hole spin. The result is shown in Fig. 6] The purpose of this exercise was to investigate whether the QNM trajectory exhibits the characteristic "looping" behaviour, first found in the studies of gravitational and electromagnetic field QNMs for Kerr and Reissner-Nordström black holes [20, 56]. Fig. 6] confirms that this behaviour is also present for scalar fields. It has been suggested [20, [56] that this peculiar behaviour is somehow associated with the change in the potential near to the horizon as the spin varies. However, since it is unclear how the high overtone modes are related to the potential a fully satisfactory explanation is still missing (see, however, [57] for an interesting point of view.

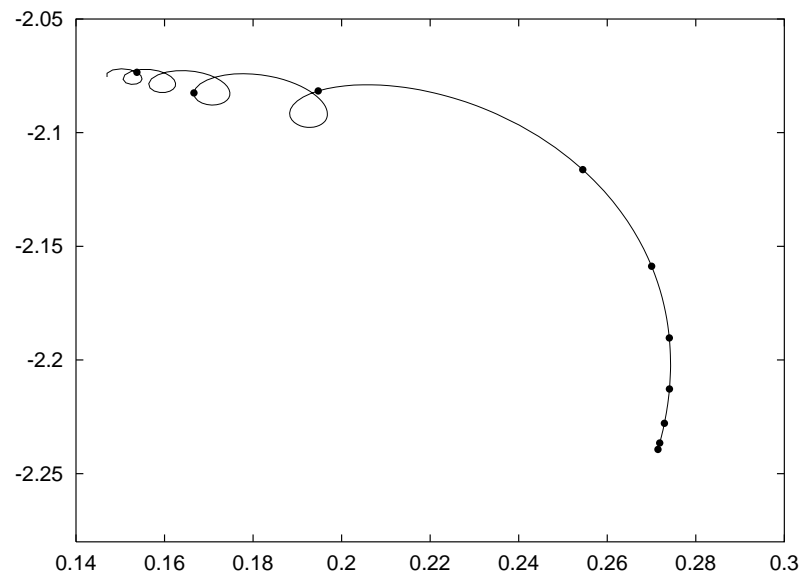

FIG. 6: The trajectory of the ninth $\ell=2, m=0$ overtone for $0 \leq a / M \leq 0.92$ (we show $\operatorname{Im} \omega_{n} M$ as a function of Re $\omega_{n} M$ ). Dots represent the position of the mode for $a / M=0,0.1, \ldots 0.9$. The characteristic "looping" motion is prominent for moderate to high spins. 


\begin{tabular}{|c|c|c|}
\hline$\omega M$ & $l_{n}$ & $r_{n}$ \\
\hline 0.500 & $2.086844+0.504735 i$ & $0.527566+0.019735 i$ \\
& $2.188325+1.479613 i$ & $0.448169-0.888761 i$ \\
& $2.342582+2.392097 i$ & $-0.350846-1.171626 i$ \\
\hline 1.000 & $4.690075+0.501236 i$ & $0.473472+0.575418 i$ \\
& $4.742988+1.494954 i$ & $2.369138-1.190595 i$ \\
& $4.839323+2.466091 i$ & $0.045487-5.147693 i$ \\
\hline 2.000 & $9.889215+0.500325 i$ & $-0.879437+0.579492 i$ \\
& $9.915868+1.498744 i$ & $3.340145+6.574819 i$ \\
& $9.967248+2.490034 i$ & $25.613332-5.812667 i$ \\
\hline
\end{tabular}

TABLE IV: The first three Schwarzschild Regge poles $\left(l_{n}\right)$ and the associated residues $\left(r_{n}\right)$ for three different (real) frequencies $\omega M$.

\begin{tabular}{|c|c|c|}
\hline$a / M$ & $m=+2$ & $m=-2$ \\
\hline 0.0 & $4.690075+0.501235 i$ & $4.690075+0.501235 i$ \\
0.1 & $4.608103+0.500711 i$ & $4.763131+0.500574 i$ \\
0.2 & $4.516055+0.498888 i$ & $4.828131+0.498790 i$ \\
0.3 & $4.412352+0.495582 i$ & $4.885722+0.495906 i$ \\
0.4 & $4.294773+0.490504 i$ & $4.936387+0.491909 i$ \\
0.5 & $4.160054+0.483194 i$ & $4.980478+0.486748 i$ \\
0.6 & $4.003103+0.472870 i$ & $5.018235+0.480332 i$ \\
0.7 & $3.815191+0.458088 i$ & $5.049802+0.472521 i$ \\
0.8 & $3.578931+0.435695 i$ & $5.075234+0.463109 i$ \\
0.9 & $3.247925+0.396177 i$ & $5.094504+0.451794 i$ \\
0.99 & $2.617843+0.278170 i$ & $5.106494+0.439626 i$ \\
\hline
\end{tabular}

TABLE V: Numerical results for the fundamental $m= \pm 2$ Kerr Regge poles $\left(l_{n}\right)$ for a selection of black hole spins. The frequency is $\omega M=1$.

\section{B. Regge poles}

As mentioned in the Introduction, Regge pole calculations are comparatively easy to perform since as the associated wavefunction (6) remains well behaved as $r_{*} \rightarrow \pm \infty$. This means that one does not have to deal with divergences, and the identification of in- and outgoing wave solutions is straightforward. For a given complex $l$, the set of equations (24) and (25) can be directly integrated along the real $r_{*}$-axis without any serious problems.

To test our numerical code we have calculated the first few Regge poles for a Schwarzschild black hole for three different frequencies. The obtained results are listed in Table IV As far as the Regge pole positions are concerned, the results are in agreement with the approximate phase-integral results given by Andersson 32. However, our calculation has confirmed an earlier suggestion by Jensen [58] that the phase-integral residues had a systematic error. As in the case of QNMs, we believe the present method is the most efficient and reliable way to calculate black-hole Regge poles.

We next consider the Regge poles of a Kerr black hole. This is a problem that, as far as we are aware, has not been discussed before. As in the case of QNMs, the loss of spherical symmetry in the background spacetime unfolds the $2 l+1$ degeneracy of the non-rotating resonances. The pole trajectories (for the first two Regge poles) are illustrated in Fig. 7 for $\omega M=1$ and $0 \leq a / M \leq 0.99$, and a sample of numerical data is given in Table $\nabla$ Figure 7 shows obvious similarities with the QNMs trajectories in figure 3 This is not surprising given the close relationship between the two classes of scattering resonances [29].

The most important conclusion we can draw from our results concerns the physical interpretation of the Kerr Regge poles. First of all, it is clear that $m>0(m<0)$ Regge poles would describe co-rotating (counter-rotating) waves. Intuitively, we would expect that the "angular life" (the inverse of Im $l_{n}$, cf. 32|) of the former would be significantly increased due to rotational frame dragging. As in the case of QNMs, we find that the $m=l$ poles are the most "sensitive" to the black hole's rotation. This strongly suggests that these poles can be associated with waves propagating around the black hole in the vicinity of the equatorial plane. More evidence to support this intuitive picture comes from the behaviour of $\operatorname{Re} l_{n}$. The decrease (increase) of this quantity, as the black hole spins up, 
is qualitatively expected from the corresponding decrease (increase) of the critical impact parameter and unstable photon orbit for prograde (retrograde) motion in Kerr spacetime.

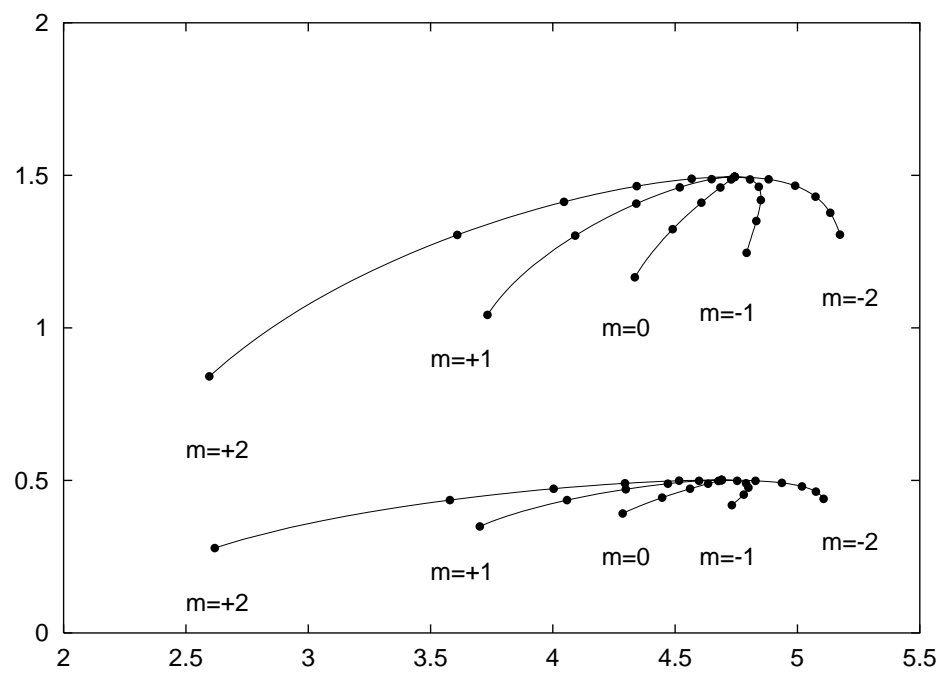

FIG. 7: The first two Kerr Regge poles (we show $\operatorname{Im} l_{n}$ as a function of $\operatorname{Re} l_{n}$ ) for various black hole rotation rates $0 \leq a / M \leq$ 0.99. Dots represent the position of each pole for $a / M=0,0.2,0.4, \ldots, 0.99$. The frequency is $\omega M=1$. Note the distinct difference between the $m>0$ and $m<0$ branches which suggests that the former (latter) can be associated with prograde (retrograde) propagating waves. There are also obvious similarities in the behaviour of Regge poles and QNMs (compare this figure with Fig. [3]

\section{CONCLUDING REMARKS}

We have developed simple numerical methods for calculating black hole resonances (QNMs and Regge poles). The basic ingredients of our schemes are (i) the use of (Prüfer) phase functions in place of the original wavefunction (ii) the integration of the resulting equations along rotated contours in the $r_{*}$-plane (when the frequency is complex), and (iii) the implementation of Müller's algorithm for locating the complex roots of $1 / \mathcal{S}(\omega, l)$ function (the inverse scattering matrix element, whose zeros define the resonances).

We have applied our schemes to the problem of calculating gravitational and massless scalar field perturbation QNMs and Regge poles for rotating and non-rotating black holes. The obtained numerical data verify all existing results in the literature with high precision. Moreover, we have provided some new results. In particular, we have shown how the slowest damped Kerr QNMs match the "long-lived" modes of a near extreme black hole which can be derived by an analytic approximation to the Teukolsky equation. This ties up some loose ends as far as the Kerr QNMs are concerned and also provides important support for using the approximate results to discuss the dynamics of near extreme black holes [21]. Our results for the excitation coefficients of Kerr QNMs add fuel to the debate concerning the detectability of gravitational waves associated with the long lived modes of a near extreme black hole. We have also provided the first results for Kerr Regge poles and discussed briefly how these results make sense given the established physical interpretation of their Schwarzschild counterparts.

In conclusion, we find that our methods can be regarded as reliable and useful, apart from (i) for very rapidly damped QNMs, and (ii) in the case of near extreme rotations $(a / M>0.999$ or so). This is, however, a small price to pay considering the profound simplicity and adaptability of the method. This is the main advantage of our approach. All other accurate schemes require a more sophisticated analysis: Leaver's continued fraction method [13] is not easy to implement given the slow convergence of the relevant series, and the complex-coordinate phase-amplitude method developed by one of us [15] makes use of much more intricate integration contours than we used in the present analysis. Finally, we should note that, even though we considered mainly the case of scalar perturbations in this paper, our method can readily be used for the more realistic case of gravitational perturbations. We therefore believe that our "quick and dirty" approach to black hole resonances may prove useful in a variety of exciting contexts. 


\section{Acknowledgments}

The work of K.G. was financially supported by the State Scholarships Foundation of Greece. N.A. is a Philip Leverhulme Prize Fellow and also acknowledges support from the EU Programme 'Improving the Human Research Potential and the Socio-Economic Knowledge Base' (Research Training Network Contract HPRN-CT-2000-00137).

[1] N. Andersson, Chapter 4 in Black-hole physics by V.P. Frolov and I.D. Novikov (Kluwer, Dordrecht 1998)

[2] C.V. Vishveshwara, Nature 227, 936 (1969)

[3] K.D. Kokkotas and B.G. Schmidt, Living Reviews in Relativity, electronic journal http://www.livingreviews.org (1999)

[4] H.-P. Nollert, Class. Quantum Grav. 16, R159 (1999)

[5] S. Chandrasekhar and S. Detweiler, Proc. Roy. Soc. (London) A 343 , 289 (1975)

[6] S. Detweiler, Astrophys. J. 225, 687 (1978)

[7] V. Ferrari and B. Mashhoon, Phys. Rev. D 30, 295, (1984)

[8] B.F. Schutz and C.M. Will, Astrophys. J. 291, L33 (1985)

[9] S. Iyer and C.M. Will, Phys. Rev. D 35, 3621 (1987)

[10] S. Iyer, Phys. Rev. D 35, 3632 (1987)

[11] E. Seidel and S. Iyer, Phys. Rev. D 41, 374 (1990)

[12] J.W. Guinn, C.M. Will, Y. Kojima and B.F. Schutz, Class. Quantum Grav. 7, L47 (1990)

[13] E.W. Leaver, Proc. R. Soc. London A 402, 285 (1985)

[14] E.W. Leaver, Phys. Rev. D 34, 384 (1986)

[15] N. Andersson, Proc. R. Soc. London A 439, 47 (1992)

[16] H.-P. Nollert and B.G. Schmidt, Phys. Rev. D 45, 2617 (1992)

[17] M. Berry and K.E. Mount, Rep. Prog. Phys. 35, 315 (1972)

[18] N. Fröman and P.O. Fröman, JWKB approximation: Contributions to the Theory, (Amsterdam: North Holland 1965)

[19] N.Andersson and S. Linnæus, Phys. Rev. D 46, 4179 (1992); N. Fröman, P.O. Fröman, N. Andersson and A. Hökback, Phys. Rev. D 45, 2609 (1991); N. Andersson, M.E. Araújo and B.F. Schutz, Class. Quantum Grav. 10, 757 (1993);

[20] H. Onozawa, Phys. Rev. D 55, 3593 (1997)

[21] K. Glampedakis and N. Andersson, Phys. Rev. D 64, 104021 (2001)

[22] S.A. Teukolsky, W.H. Press, Astrophys. J. 193, 443 (1974)

[23] E. Berti and K.D. Kokkotas, Asymptotic quasinormal modes of Reissner-Nordström and Kerr black holes, preprint hep-th/0303029

[24] H.-P. Nollert, Phys. Rev. D 47, 5253 (1993)

[25] N. Andersson, Class. Quantum Grav. 10, L61 (1993)

[26] O. Dreyer, Quasinormal Modes, the Area Spectrum, and Black Hole Entropy preprint gr-qc/gr-qc/0211076

[27] L. Motl, An analytical computation of asymptotic Schwarzschild quasinormal frequencies, preprint gr-qc/0212096

[28] S. Hod, Phys. Rev. Lett. 81, 4293 (1998)

[29] Y. Décanini, A. Folacci and B.P. Jensen, Complex angular momentum in black hole physics and the quasi-normal modes preprint gr-qc/0212093

[30] H.M. Nussenzveig, Diffraction effects in Semiclassical Scattering (Cambridge: Cambridge University Press 1992)

[31] N. Andersson and K-E. Thylme, Class. Quantum Grav. 11, 2991 (1994)

[32] N.Andersson, Class. Quantum Grav. 11, 3003 (1994)

[33] S. Chandrasekhar and V. Ferrari, Proc. R. Soc. London A 437, 133 (1992)

[34] K. Glampedakis and N. Andersson, Class. Quantum Grav. 18, 1939 (2001)

[35] J.R. Taylor, Scattering Theory (New York:Willey, 1972)

[36] S. Chandrasekhar, The Mathematical Theory of Black Holes (Oxford University Press, New York, 1983)

[37] S.A. Teukolsky, Astrophys. J. 185, 635 (1973)

[38] B.P. Jensen, Annales De Physique 16, 117 (1989)

[39] P.D. Lax and R.S. Phillips, Scattering Theory (Academic, New York, 1967)

[40] N. Andersson, Phys. Rev. D 55468 (1997)

[41] R.A. Matzner, J. Math. Phys. 9, 163 (1968)

[42] F.A. Handler and R.A. Matzner, Phys. Rev D 22, 2331 (1980)

[43] W.E. Milne, Phys. Rev. 35, 863 (1930)

[44] W.I. Newman and W.R. Thorson, Phys. Rev. Lett. 29, 1350 (1972); Can. J. Phys. 50, 2997 (1972)

[45] J.D.Pryce, Numerical Solution of Sturm-Liouville Problems (Oxford Science Publications, Oxford, England, 1993)

[46] P. Pajunen, J. Chem. Phys. 88, 4268, (1988)

[47] P. Pajunen, J. Comp. Phys. 82, 16, (1989)

[48] N. Andersson, J. Phys. A: Math. Gen. 26, 5085 (1993)

[49] N. Andersson, K.D. Kokkotas and B.F. Schutz, Mon. Not. R. Astron. Soc. 274, 1039 (1995)

[50] M.E. Araújo, D. Nicholson and B.F. Schutz, Class. Quantum Grav. 10, 1127 (1993)

[51] W.H.Press, S.A. Teukolsky, W.T. Vetterling and B.P. Flannery, Numerical Recipes (Cambridge University Press, Cam- 
bridge, England, 1992)

[52] M. Sasaki and T. Nakamura. Prog. Theor. Phys. 67, 1788 (1982)

[53] N. Andersson Phys. Rev. D 51353 (1995).

[54] L.S. Finn Phys. Rev. D 465236 (1992).

[55] N. Andersson, Proc. R. Soc. London A 442, 427 (1993)

[56] N. Andersson and H. Onozawa, Phys. Rev. D 54, 7470 (1996)

[57] H. Liu, Class. Quantum Grav. 12, 543 (1995)

[58] B.P. Jensen, private communication 